\title{
1 Detection and treatment strategy for Tritrichomonas muris in the common laboratory
}

\section{2 mouse}

5 Andreia S. Da Costa ${ }^{1,2}$, Tisha M. Graham ${ }^{2}$, Jennifer A. Duncan ${ }^{3}$, Smitha P.S. Pillai ${ }^{3}$ and Jennifer

6 M. Lund Lu $^{1,2,4}$

$9 \quad{ }^{1}$ Graduate Program in Pathobiology, Department of Global Health, University of Washington,

10 Seattle, WA

$11{ }^{2}$ Vaccine and Infectious Disease Division, Fred Hutchinson Cancer Research Center, Seattle,

$12 W A$

$13{ }^{3}$ Comparative Medicine, Fred Hutchinson Cancer Research Center, Seattle, WA

\section{$14{ }^{4}$ Lead Contact}

15

16 Running Title: Treating T. muris in lab mice

18 Corresponding Author and Lead Contact:

19 Jennifer M. Lund

20 Fred Hutchinson Cancer Research Center

211100 Fairview Ave N, E5-110

22 Seattle, WA, 98109 U.S.A.

23 Phone number: 206-667-2217 
24 Fax Number: 206-667-7767

25 E-mail: jlund@ fredhutch.org

26

27 Conflict of interest

28 The authors declare no conflicts of interest.

29

$30 \quad$ Funding

31 Funding for the present study was provided by the National Institutes of Allergy and Infectious

32 Diseases of the US National Institutes of Health (R01 AI087657 and R01 AI141435 JML). In

33 addition, ASC was supported by the Diseases of Public Health Importance Training Grant

34 (T32AI007509).

35

36 


\section{Summary}

38 Maintaining a specific pathogen-free (SPF) mouse colony is critical to avoid potentially

39 confounding variables introduced by unknown infections. Here, we report an instance of

40 protozoan Tritrichomonas muris (T. muris) infection of mice within an SPF facility. Although $T$.

41 muris has been considered a commensal organism, we observed instances of asymmetric

42 infection in gene-knockout mice as compared to wild-type mice. We utilized treatment with

43 metronidazole and confirmed successful elimination of T. muris from our SPF colony using

44 extraction of fecal DNA followed by PCR detection. We propose that T. muris testing should be

45 considered for SPF mice, particularly in immunity studies.

46

\section{Keywords}

48 Tritrichomonas muris; SPF mice; gene knock-out mice; pathosymbiont; protozoan infection 


\section{Introduction}

50 Tritrichomonas muris is a triflagellate single-celled protozoan that infects the intestinal

51 tissues of mice and other rodents. It is closely related to Tritrichomonas foetus, a pathogen

52 known to infect bovine reproductive organs (Yao and Koster, 2015). T. muris, however, has been

53 listed as a commensal organism (Baker, 2008) and is therefore rarely screened for during routine

54 mouse colony maintenance. Nevertheless, a proteomic analysis of intestinal tissue collected from

55 mice infected with $T$. muris differentially expressed several proteins compared to uninfected

56 intestines, a subset of which were immune related (Kashiwagi et al., 2009), suggesting that $T$.

57 muris infections may trigger an immune response. More recently, a study of T-cell-driven colitis

58 identified that $T$. muris infection exacerbates disease and skews baseline T-cell homeostasis

59 toward a more pro-inflammatory mucosal environment (Escalante et al., 2016), and a second

60 study also found that host-protozoan interactions can alter mucosal immune homeostasis

61 (Chudnovskiy et al., 2016). T. muris infection has been shown to increase the frequency of

62 intestinal tuft cells which can induce IL-13 secretion from intestinal innate lymphoid cells (ILC)

63 (Howitt et al., 2016; von Moltke et al., 2016). Additional studies showed that a metabolite from

64 T. muris trigger tuft cell production of IL-25 (Schneider et al., 2018).

During a routine intestinal tissue preparation, we discovered that a subset of our mouse

66 colony was infected with T. muris. Specifically, we determined that a conditional knock-out

67 (cKO) mouse strain was most affected, and so it was critical that we eliminate the potential

68 confounding effects of a T. muris infection in our cKOs for accurate comparison to WT controls.

69 Thus, we developed a PCR-based detection assay to determine the T. muris infection status of

70 our cKO mouse model in conjunction with a metronidazole-based treatment plan that allowed us

71 to re-establish the SPF status of our colony. 


\section{Results}

74 T. muris infection can potentially confound characterization of novel immune system-associated

75 gene deletion mouse models.

Gene knockout (KO) and Cre/lox conditional knockout (cKO) mouse models have

78 proven to be invaluable tools in the reductive investigation of individual gene functions in

79 specific cells and tissues. Use of KOs in studies of the immune system vary widely and have

80 helped elucidate the inner workings of immune cells as well as their relationships with other

81 systems within the organism (Mak et al., 2001). Accordingly, we set out to characterize the

82 effect of integrin $\beta 1$ deletion in regulatory $\mathrm{T}$ cells (Treg) using Itgb $1^{\text {flox/flox }} \times$ Foxp $3^{\text {Cre }}(\mathrm{cKO})$

83 mice (Da Costa et al., 2019). When preparing small intestine from cKO and WT control mice for

84 the analysis of lamina propria lymphocytes, we observed a dramatic discoloration in the cKO-

85 derived tissue (Fig. 1A) and subsequently detected a free-swimming organism in the tissue

86 supernatant under 20X magnification. Fecal samples from cKO mice were sent for identification

87 of the protozoan (IDEXX Bioresearch, Columbia, MO, USA), which was confirmed to be

88 Tritrichomonas muris. Although T. muris has been grouped with other protozoan infections

89 associated with symptoms including weight loss, runting and diarrhea (Charles River

90 Laboratories International, 2009), we did not find a statistically significant weight difference in

$91 \mathrm{cKO}$ mice as compared to age-matched WT controls (Fig. 1B). To confirm infection, we

92 performed histopathological evaluation of sections of the gastrointestinal tract. Within the lumen

93 of cecum and colon in the cKO group, there were large numbers of oval to pyriform protozoal

94 trophozoites, measuring approximately 20x10 $\mu \mathrm{m}$, with eosinophilic cytoplasm and round 
95 hyperchromatic nuclei consistent with Trichomonas species (Fig. 1C, left panel). No changes in

96 mucosal inflammation were detected. In the control mouse (Fig. 1C, right panel), no protozoa

97 were detected. Spleen sections were also evaluated from both groups, though we found no

98 appreciable histological differences between T. muris-infected cKO mice and uninfected WT

99 controls (Fig. 1D). Altogether, we found evidence of select T. muris infection of the intestine of a

100 cKO mouse strain compared to WT mice within an SPF mouse colony. 

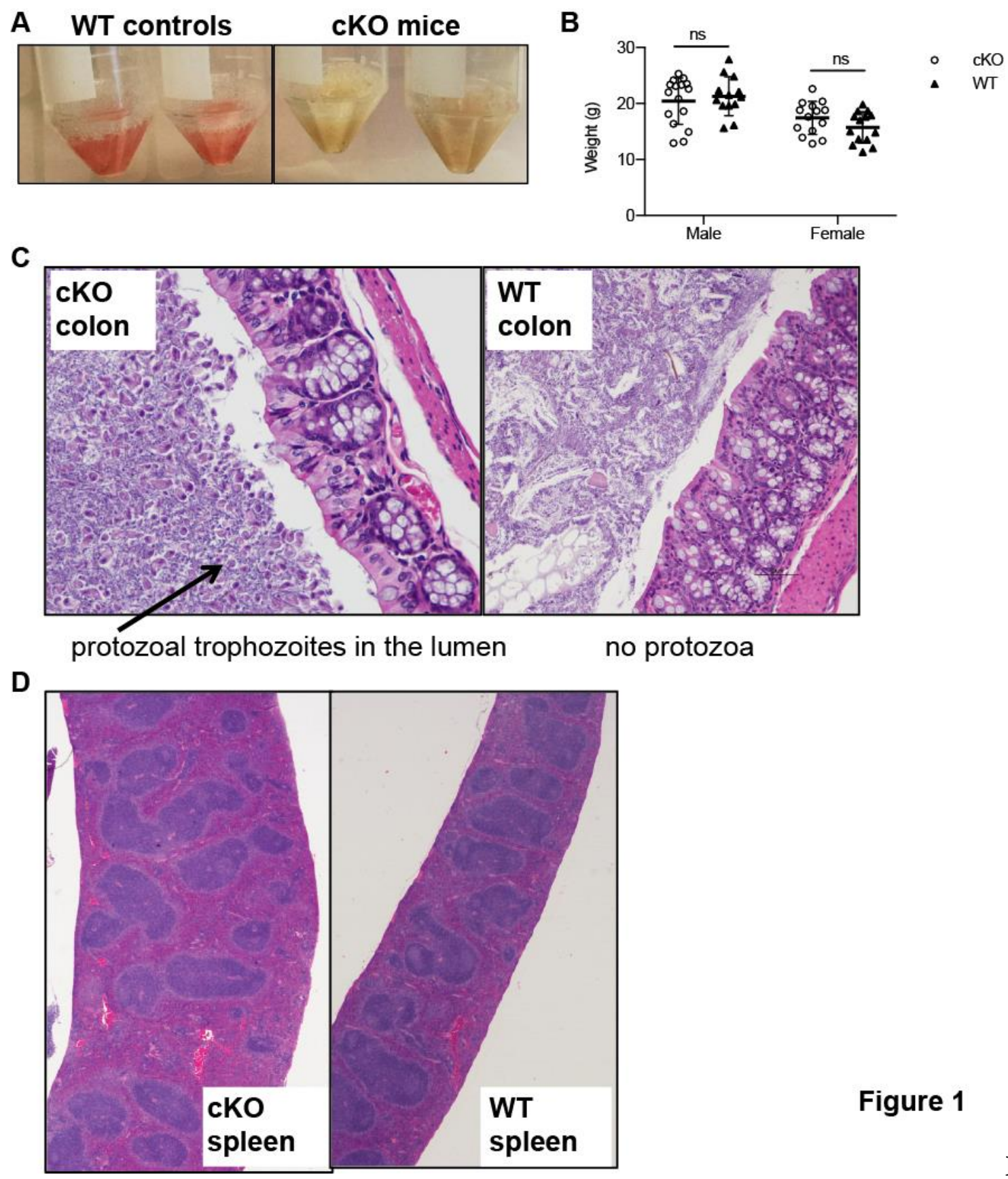

Figure 1

103 1. T. muris infection can confound characterization of novel immune system-associated gene

104 deletion mouse models. (A) Left panel shows excised and sectioned small intestine from two

105 adult C57BL/6J male mice in 1X RPMI media supplemented with 5\% heat inactivated fetal

106 bovine serum (HI-FBS) after incubation at $37^{\circ} \mathrm{C}$ with agitation at $220 \mathrm{rpm}$ for $30 \mathrm{mins}$ in EDTA

107 Buffer. Original image contrast has been increased by $40 \%$ to improve visibility. Right panel 
108 shows small intestine tissue after processing as in left panel, however tubes contain tissue from

109 adult $\mathrm{cKO}$ that are infected with T. muris. (B) Weights were recorded from male and female cKO

110 and WT on C57BL/6J background. Average weight/age of cKO (n=16) males: 20.4g/52.6 dys,

111 cKO (n=14) females: $17.4 \mathrm{~g} / 48$ dys; WT ( $\mathrm{n}=13)$ males: $21.3 \mathrm{~g} / 53.2$ dys, WT $(\mathrm{n}=14)$ females:

$112 \quad 15.7 \mathrm{~g} / 48.4$ dys. Plots display range and median for each group. Statistics represent two-stage

113 linear step-up procedure of Benjamini, Krieger and Yekutieli, with $\mathrm{Q}=1 \%$. Comparison groups

114 (cKO vs. WT) were analyzed individually, without assuming a consistent SD. (C)

115 Histopathological evaluation of colon from cKO or WT mice. Arrow in left panel indicates

116 protozoal trophozoites which are absent in colon tissue assessed from WT mice in the right

117 panel. (D) Histopathological evaluation of spleens from cKO and WT mice. No gross or

118 histological differences were noted between infected cKO and uninfected WT mice. 
PCR-based detection of T. muris reveals wide-spread but not uniform infection of SPF mice.

124 sequence data for T. muris, we aligned the $18 \mathrm{~s}$ ribosomal sequences of T. muris and T. foetus

125 using the nucleotide blast program hosted by NCBI BLAST, and selected primers that covered

126 conserved exon regions shared between both species. Using these primers for PCR, we sought to

127 determine the extent of T. muris infection within our cKO breeder population and colony. To

128 ensure that infection status was attributed correctly to individual mice, each mouse tested was

129 segregated to a clean receptacle until a fresh fecal sample was produced. We found that each

130 cKO breeder cage sampled tested positive for T. muris DNA (Fig. 2A lanes 3-10). Furthermore,

$131 \operatorname{Itgb}^{\text {flox/wt }} \times$ Foxp3 ${ }^{\text {Cre }}$ mice also tested positive (Fig. 2A, lanes 1 and 2). This indiscriminate

132 infection pattern suggests that the gene deletion in the cKO mice did not result in an immune

133 deficit that made them uniquely susceptible to T. muris. Another cKO strain, CTLA4 ${ }^{\text {flox } f l o x} \mathrm{x}$

134 Foxp3 $3^{\text {Cre }}$, was previously tested commercially and so was utilized successfully as a negative

135 infection-control (Fig. 2A, lanes 11 and 12). After determining complete infection of Itgb $1^{\text {flox/flox }}$

$136 \mathrm{x}$ Foxp3 $3^{\text {Cre }}$ breeding stock, we further confirmed that the infection had spread to other strains

137 despite the lack of physical contact (Fig. 2A, lanes 14-20). Surprisingly, the sentinel mice housed

138 in the cKO rack tested negative for T. muris DNA (Fig. 2A, lane 21). As sentinel mice are

139 routinely exposed to bedding from various cages housed in the same rack, therefore the lack of $T$.

140 muris, infection in these mice underscores the need to expose sentinels to fresh feces to most

141 effectively surveil a colony. 


\section{Figure 2}

A

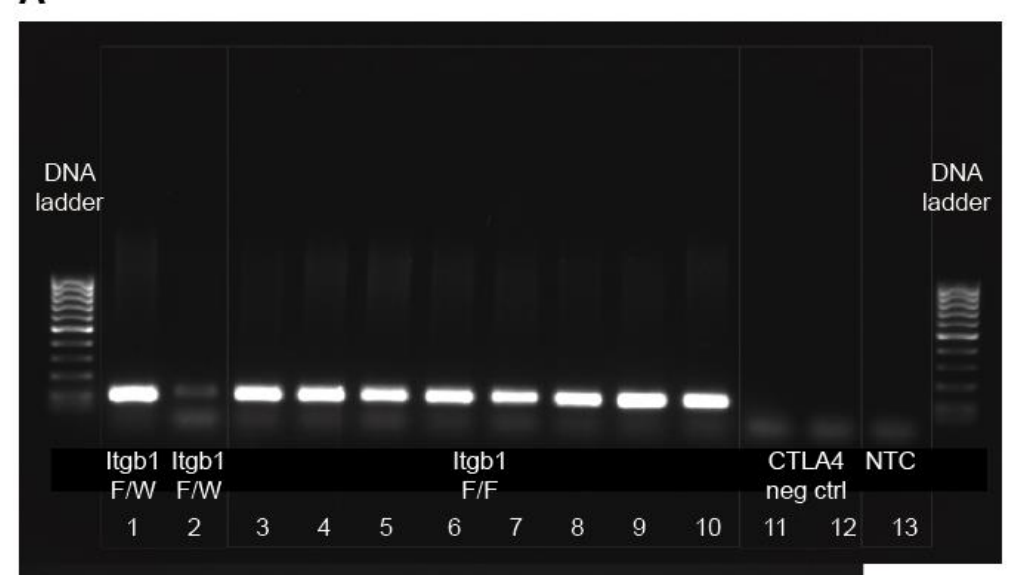

144

Figure 2. T. muris specific primer selection and PCR-based detection of T. muris infection.

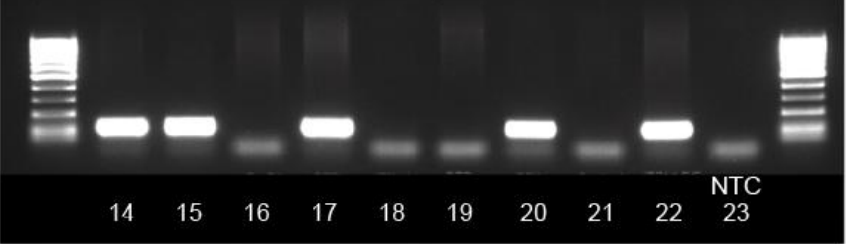

(A) Using primers and PCR protocol described, we collected fecal samples from all breeder cages housing our Itgb $1^{\mathrm{F} / \mathrm{F}} \times$ Foxp $3^{\mathrm{Cre}}$ and Itgb $1^{\mathrm{F} / \mathrm{W}} \times$ Foxp $3^{\mathrm{Cre}}$ control mice and found that all breeder cages were positive for presence of $T$. muris DNA (lanes 1-10). CTLA4 (Ctla4 ${ }^{\mathrm{F} / \mathrm{F}} \mathrm{x}$ Foxp $3^{\mathrm{Cre}}$ ) are a mouse strain known to be negative through commercial testing (IDEXX Bioresearch, Inc) (lanes 11-12). NTC = non-template control where template has been replaced with additional DNase-free $\mathrm{dH}_{2} \mathrm{O}$ (lane 13). Additional strains within our colony were tested and some were found to be infected (lanes 14-20). Long-lived sentinel mice associated with the

154 T. muris DNA after metronidazole treatment (lanes 1-2). + control = DNA extracted from fecal

155 samples of CKO mice prior to treatment (lane 3). NTC = No template control (lane 4). 
Metronidazole treatment in combination with a fostering plan can clear T. muris infection

To avoid the costly and time-consuming, albeit guaranteed strategy of replacement of all

159 affected cages by purchase of new breeder stock, we used a combination of metronidazole

160 treatment in combination with a pup-fostering plan. Metronidazole serves as both an antibiotic

161 and antiprotozoal medication often prescribed to treat human Giardia lamblia, Clostridium

162 difficile and Trichomonas vaginalis infections (Freeman et al., 1997). Although very little has

163 been published describing treatment strategies for T. muris, Roach et al showed that T. muris

164 introduced from infected gerbils to mouse hosts was successfully treated by supplementing

165 drinking water with metronidazole or tinidazole (Roach et al., 1988).

166 Thus, we purchased outbred Swiss Cr1:CD1 (ICR) breeders both for ease of

167 differentiation of foster pups by coat color, and due to anecdotal reports of CD-1 dams' efficient

168 handling of large litter sizes. CD-1 mice were tested upon arrival and found to be negative for

169 fecal presence of T. muris DNA. CD-1 and cKO breeder pairs were subsequently set-up on

170 different racks. After visual confirmation of the development of a mucus plug in pregnant

171 females, breeder males were removed from the breeding cages. Gestating dams were then

172 monitored closely and metronidazole-supplemented water introduced approximately 5 days prior

173 to expected birth. Upon birth, CD-1 pups were removed from the breeder cage and replaced with

174 the recently birthed cKO pups. Metronidazole supplementation was continued for 5 days post-

175 pup transfer. After a total of 10 days of water supplementation, metronidazole treatment was

176 ceased and pups remained under the care of the CD-1 foster dam until 21 days post-birth. Foster

177 pups were weaned, fecal samples collected and DNA extracted. PCR analyses of the collected

178 fecal samples were negative for the presence of T. muris DNA, indicating successful treatment 
179 (Fig. 2B). Although several studies, both mouse and human, have concluded that metronidazole

180 does not increase the incidence of cancer in treated patients (or animals) (Chacko and Bhide,

181 1986; Thapa et al., 1998), the Food and Drug Administration (FDA) has labeled metronidazole

182 as a potential mutagen and teratogen. Thus, we completed several rounds of backcrossing of the

183 newly fostered T. muris-free cKO mice to WT C57BL/6J mice, both to limit the carry-over of

184 potential mutagenic effects of metronidazole treatment and to mitigate the effects of genetic

185 bottlenecking as a result of the necessarily small number of pups fostered. Taken, together, we

186 report a strategy to test and treat for T. muris infection in mice. 


\section{Discussion}

We describe a treatment strategy developed in response to the incidental finding of $T$.

191 muris infection within select strains in our mouse colony. To ensure the accurate and high-

192 throughput identification of the protozoa, we developed a PCR-based assay to evaluate the

193 presence of $T$. muris DNA in fresh fecal samples. Through this testing, we determined that the

194 infection had spread through the colony and that the use of sentinel mice was not sufficient to

195 detect T. muris infection. Due to our finding of differential T. muris infection of the intestine

196 between cKO and WT mice (Figs. 1C and 2A) in which we wished to analyze immune cell

197 phenotypes, along with the knowledge that Tritrichomonas infections have a demonstrated effect

198 of altering mucosal immune homeostasis (Escalante et al., 2016; Howitt et al., 2016; Schneider et

199 al., 2018; von Moltke et al., 2016) that could thus confound our studies, we elected to eradicate

200 the infection from our colony. We developed a treatment plan that resulted in the successful

201 restoration of the original controlled SPF-status of our cKO mice, thereby eliminating a potential

202 driver of differential immune homeostasis between mouse strains.

The regulated exposure of experimental lab animals to a pre-determined list of pathogens

204 and commensals has become a hallmark of rigorously conducted studies. Ensuring the reliability

205 and integrity of data generation within animal models requires a thorough understanding of

206 potential confounding variables. This is especially important in studies that propose to

207 investigate the complex subtleties of a developing immune response. Furthermore, studies that

208 include the characterization of a novel gene deletion in a mouse model are particularly

209 vulnerable to misdirection by unknown confounding variables. As such, it is critical that

210 investigators remain vigilant of the microbial status of their colonies as well as remaining open 
211 to the re-evaluation of micro-organisms that may have been deemed commensals in the past.

212 Based on our study, we propose that T. muris should be monitored as a pathosymbiont in SPF

213 colonies, particularly if mice are to be used for the study of immunity, since infection of a KO

214 but not a control could lead to a mis-classification or mis-interpretation of any differential data. 


\section{Methods}

218 Ethics Statement

219 All animal work was approved by the Fred Hutchinson Cancer Research Center (Fred Hutch)

220 and treatment provided through the Comparative Medicine department. The Office of Laboratory

221 Animal Welfare of the National Institutes of Health has approved Fred Hutch (\#A3226-01), and

222 this study was carried out in strict accordance with the Public Health Service Policy on Humane

223 Care and Use of Laboratory Animals.

225 T. muris specific PCR amplification and visualization

226 Fresh fecal pellets were collected from mice and fecal DNA extracted using the ZR Fecal DNA

227 MiniPrep ${ }^{\mathrm{TM}}$ kit (Zymo Research Corp., CA, U.S.A.). T. muris forward and reverse primers were

228 purchased through Integrated DNA Technologies (IDTDNA.com). Primer sequences are as

229 follows: forward primer, GCAATGGATGTCTTGGCTTC, and reverse primer,

230 GCGCAATATGCATTCAAAGA. Master mix composition was based on manufacturer's

231 recommendations included in the 2X MyTaq ${ }^{\text {TM }}$ HS Red Mix purchased through Bioline USA

232 Inc, (Taunton, MA). Annealing temperature, duration and cycle number were determined

233 empirically: $\mathrm{Tm}(50 \mathrm{mM} \mathrm{NaCl})$ for forward primer $=54.4^{\circ} \mathrm{C}$; $\mathrm{Tm}(50 \mathrm{mM} \mathrm{NaCl})$ for reverse

234 primer $=51.9^{\circ} \mathrm{C}$. Gel electrophoreses of PCR products were done using $1 \%$ Agarose gel matrix

235 with the addition of 10,000X GelRed ${ }^{\mathrm{TM}}$ Nucleic Acid Gel Stain (Biotium, Inc. CA, USA).

236 Samples were run alongside 100bp exACTGene ${ }^{\mathrm{TM}}$ DNA ladder (Fisher BioReagents ${ }^{\mathrm{TM}}$ ) for

$237 \sim 35 \mathrm{mins}$ at $100 \mathrm{~V}$ in $1 \mathrm{X}$ TAE Buffer. Expected PCR product is approximately 65bp in length. 
Metronidazole treatment

240 An optimized dose of $2.5 \mathrm{mg} / \mathrm{ml}$ metronidazole in a $1 \%$ sucrose solution was used to treat $T$.

241 muris in mice(Roach et al., 1988). A solution of $2.5 \mathrm{mg} / \mathrm{ml}$ metronidazole was prepared by

242 crushing a $250 \mathrm{mg}$ metronidazole tablet and dissolving it in $100 \mathrm{ml}$ of a $1 \% \mathrm{w} / \mathrm{v}$ sucrose solution.

243 The solution was then passed through a $22 \mathrm{um}$ filter and placed in sterile water bottles wrapped in

244 foil to prevent photo-degradation.

246 Mice

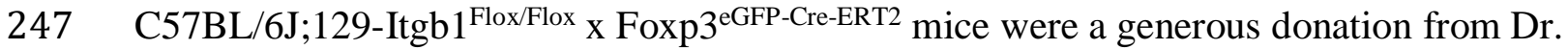

248 Alexander Rudensky (Memorial Sloan Kettering Cancer Center) and have been maintained at the

249 Fred Hutch Animal Facility for 10+ years. Six-week old Crl:CD1 (ICR) foster dams were

250 purchased from Charles River.

252 Histopathology

253 Complete necropsies were performed on wild-type (WT) and cKO mice. Tissues including

254 spleen, liver, and intestines were collected and fixed in 10\% neutral buffered formalin, routinely

255 processed and embedded in paraffin, sectioned at $4 \mu \mathrm{m}$, and stained with hematoxylin and eosin

256 for histological evaluation. 


\section{Acknowledgements}

259 The authors thank Rajesh Uthamanthil, DVM, MVSc, PhD, DACLAM for advice and the Fred

260 Hutchinson Cancer Research Center Dept. of Comparative Medicine for assistance and guidance.

\section{Author Contributions}

263 Development and execution of treatment strategy (A.S.D., T.M.G, J.A.D.); Histopathology

264 scoring, interpretation and analysis (S.P.S.P); Writing and preparation of manuscript (A.S.D. and

265 J.M.L.); Critical revisions and guidance (J.M.L).

266

\section{Declaration of Interests}

268 The authors declare that they have no competing interests.

\section{Corresponding author}

270 Correspondence to Jennifer M. Lund (jlund@ fredhutch.org). 


\section{References}

274 Baker, D.G. (2008). Parasites of rats and mice, in Flynn's Parasites of Laboratory Animals.

275 Blackwell Publishing Ltd Second Edition, 303-397.

276 Chacko, M., and Bhide, S.V. (1986). Carcinogenicity, perinatal carcinogenicity and

277 teratogenicity of low dose metronidazole (MNZ) in Swiss mice. J Cancer Res Clin Oncol 112,

$278 \quad 135-140$.

279 Charles River Laboratories International, I. (2009). Intestinal Protozoa in Rodents: Technical

280 Sheet. Charles River Research Models and Services, 1-2.

281 Chudnovskiy, A., Mortha, A., Kana, V., Kennard, A., Ramirez, J.D., Rahman, A., Remark, R.,

282 Mogno, I., Ng, R., Gnjatic, S., et al. (2016). Host-Protozoan Interactions Protect from Mucosal

283 Infections through Activation of the Inflammasome. Cell 167, 444-456 e414.

284 Da Costa, A.S., Graham, J.B., Swarts, J.L., and Lund, J.M. (2019). Regulatory T cells limit

285 unconventional memory to preserve the capacity to mount protective CD8 memory responses to

286 pathogens. Proc Natl Acad Sci U S A.

287 Escalante, N.K., Lemire, P., Cruz Tleugabulova, M., Prescott, D., Mortha, A., Streutker, C.J.,

288 Girardin, S.E., Philpott, D.J., and Mallevaey, T. (2016). The common mouse protozoa

289 Tritrichomonas muris alters mucosal T cell homeostasis and colitis susceptibility. J Exp Med

$290213,2841-2850$.

291 Freeman, C.D., Klutman, N.E., and Lamp, K.C. (1997). Metronidazole. A therapeutic review and 292 update. Drugs 54, 679-708. 
293 Howitt, M.R., Lavoie, S., Michaud, M., Blum, A.M., Tran, S.V., Weinstock, J.V., Gallini, C.A.,

294 Redding, K., Margolskee, R.F., Osborne, L.C., et al. (2016). Tuft cells, taste-chemosensory cells,

295 orchestrate parasite type 2 immunity in the gut. Science 351, 1329-1333.

296 Kashiwagi, A., Kurosaki, H., Luo, H., Yamamoto, H., Oshimura, M., and Shibahara, T. (2009).

297 Effects of Tritrichomonas muris on the mouse intestine: a proteomic analysis. Exp Anim 58,

$298 \quad 537-542$.

299 Mak, T.W., Penninger, J.M., and Ohashi, P.S. (2001). Knockout mice: a paradigm shift in

300 modern immunology. Nat Rev Immunol 1, 11-19.

301 Roach, P.D., Wallis, P.M., and Olson, M.E. (1988). The use of metronidazole, tinidazole and

302 dimetridazole in eliminating trichomonads from laboratory mice. Lab Anim 22, 361-364.

303 Schneider, C., O'Leary, C.E., von Moltke, J., Liang, H.E., Ang, Q.Y., Turnbaugh, P.J.,

304 Radhakrishnan, S., Pellizzon, M., Ma, A., and Locksley, R.M. (2018). A Metabolite-Triggered

305 Tuft Cell-ILC2 Circuit Drives Small Intestinal Remodeling. Cell 174, 271-284 e214.

306 Thapa, P.B., Whitlock, J.A., Brockman Worrell, K.G., Gideon, P., Mitchel, E.F., Jr., Roberson,

307 P., Pais, R., and Ray, W.A. (1998). Prenatal exposure to metronidazole and risk of childhood

308 cancer: a retrospective cohort study of children younger than 5 years. Cancer 83, 1461-1468.

309 von Moltke, J., Ji, M., Liang, H.E., and Locksley, R.M. (2016). Tuft-cell-derived IL-25 regulates

310 an intestinal ILC2-epithelial response circuit. Nature 529, 221-225.

311 Yao, C., and Koster, L.S. (2015). Tritrichomonas foetus infection, a cause of chronic diarrhea in

312 the domestic cat. Vet Res 46, 35. 